\title{
sciendo
}

\section{On Phenomenal Functionalism about the Properties of Virtual and Non-virtual Objects}

\author{
Alyssa Ney \\ University of California, Davis \\ DOI: $10.2478 /$ disp-2019-0005 \\ BIBLID [0873-626X (2019) 55; pp.399-410]
}

\begin{abstract}
According to phenomenal functionalism, whether some object or event has a given property is determined by the kinds of sensory experiences such objects or events typically cause in normal perceivers in normal viewing conditions. This paper challenges this position and, more specifically, David Chalmers's use of it in arguing for what he calls virtual realism.
\end{abstract}

\section{Keywords}

Virtual reality, virtual objects, phenomenal functionalism, secondary qualities.

\section{Introduction}

In arguing for his virtual realism, that objects in virtual worlds really exist, that events in virtual worlds really happen, and that experiences in virtual reality are non-illusory, David Chalmers makes use of a way of thinking about the properties of objects and events, one we may call phenomenal functionalism. According to this view, whether some object or event has a given property is determined by the kinds of sensory experiences such objects or events typically cause in normal perceivers in normal viewing conditions. Chalmers suggests that phenomenal functionalism is plausible both for the properties of virtual and of non-virtual objects and events. It is a view he suggests is plausible for what philosophers have traditionally thought of both as secondary qualities, for example, color properties, and primary qualities, for example, spatial properties like shapes and sizes. This 
wide-sweeping phenomenal functionalism allows Chalmers to make the case that virtual objects and events not only really exist or happen, but that they exist and happen in a way that is on a par with the way non-virtual objects and events exist or happen, and that they have the kinds of properties non-virtual entities seem to have: colors, shapes, and sizes.

In this discussion, my goal will be to probe the plausibility of phenomenal functionalism with respect to each of the four kinds of property: color properties in virtual realities, color properties in non-virtual realities, spatial properties in virtual realities, and spatial properties in non-virtual realities. After explaining Chalmers's view in more detail, I will raise considerations suggesting that while phenomenal functionalism may seem a natural way to understand the examples Chalmers uses of color properties and perhaps other sensory qualities like tastes and smells, this view is much less plausible when it comes to spatial properties. If this is right, then Chalmers cannot use phenomenal functionalism to defend the claim that in general, the properties of virtual objects and events are on a par with those of non-virtual objects and events. Perhaps this argument for realism ought to be confined to the sensory qualities of virtual objects.

In the section that follows, I raise considerations suggesting that whatever is to be said about phenomenal functionalism for the properties of non-virtual objects and events, such a framework is less plausible as a means of understanding the properties of virtual objects and events. And so if phenomenal functionalism is plausible for the properties of the non-virtual, this cannot help us establish any conclusions about the virtual.

In what follows, I consider what this implies for virtual realism. My sense is that in fact the argument I present in Section 3 does not provide reason to doubt virtual realism so long as one can argue there is some sense in which the spatial properties of virtual and nonvirtual objects are similar in kind. However, the most natural way of doing so and supporting virtual realism by appeal to a more plausible view about the nature of spatial properties does not seem to work. And so to save virtual realism, one must seek out some third account of spatial properties. Although the argument presented in Section 3 is in this way tentative, the argument in Section 4, against phenomenal functionalism for virtual properties, does seem to raise more of 
a threat for virtual realism, lending support to what Chalmers takes to be the main rival to his virtual realism, virtual fictionalism, as I will explain.

\section{Phenomenal functionalism and the case for virtual realism}

As Chalmers notes, an orthodox view about color properties is that red things are what cause red experiences in normal perceivers in normal circumstances. In this and other work (e.g. Chalmers 2012), he proposes we extend this view to spatial properties as well, such as shapes, sizes, and lengths. This implies round things are what cause round experiences, six feet tall things are what cause six feet tall experiences, and so on.

This immediately leads to a puzzle for his virtual realism and the view that our experiences of virtual objects are generally non-illusory. For to establish that virtual objects are real, Chalmers proposes we identify them with digital objects.

Suppose I am playing Gun Club VR on the Oculus Rift. In the game, I select a black Glock pistol. The virtual fictionalist will claim that this pistol is a fictional object. It doesn't really exist. It only exists in the fiction of Gun Club. But Chalmers notes that there is no reason to deny the reality of this virtual object so long as we see it as ultimately constituted out of the computational processes that make up the game. In general, virtual objects then are digital objects, according to Chalmers. They are data structures. Different virtual objects and events in virtual worlds correspond to different such data structures. I take no issue with this proposal.

Here is the puzzle. As I have the Oculus headset on and look down, I see that in the game, I am wearing a red shirt and have a large, round belly that makes it hard to see and access the ammunition on my belt. According to Chalmers, the shirt my game avatar wears is a digital object, a data structure. And so it is something constituted out of some bit of computer hardware. But this hardware is itself not something that causes red experiences in normal perceivers in normal viewing conditions. Opening up my Oculus in normal viewing conditions to visually access this hardware would likely not cause me to have red experiences at all.

Chalmers's response to this puzzle is to concede that my avatar's 
shirt is not red in the way non-virtual objects like ripe strawberries and stop signs are. Rather, it is another kind of red. It is red in the sense that it causes red experiences in normal perceivers under viewing conditions that are normal for this virtual reality. If any normal perceiver were in the sort of viewing conditions I am in when I look down at my shirt in the game wearing my Oculus headset, they would also have red experiences.

Mutatis mutandis for spatial properties. My (or my avatar's) belly looks round when I am playing Gun Club VR and it really is round. But it is not round in the same way that non-virtual tomatoes and baseballs are round. My avatar's belly, as a digital object, likely doesn't cause round experiences in normal viewing conditions. Rather it causes round experiences in conditions that are normal for the Gun Club virtual reality. And in this way, it is virtually round, even if not non-virtually round. ${ }^{1}$

Chalmers thus endorses a phenomenal functionalism about both colors and spatial properties both in non-virtual and virtual reality settings. This is a functionalism because whether an object is red or round is determined by the object's causal role. It is a phenomenal functionalism because whether an object is red or round is determined by the object's role in causing phenomenal experiences in certain viewing conditions.

So, according to Chalmers, the virtual colors and spatial properties of virtual objects are not the same as the non-virtual colors and spatial properties of non-virtual objects. Virtual red is a different property than non-virtual red. But that virtual and non-virtual red, as well as virtual and non-virtual round are in a relevant respect similar, or fall under a common genus, does seem crucial for his argument for virtual realism, in particular, the part of the view that says experiences in virtual reality are non-illusory. For in decent virtual realities, the digital objects we interact with look like objects we interact with outside of virtual reality settings. It is fine if the red of my avatar's shirt doesn't look just like the red of my non-virtual

\footnotetext{
${ }^{1}$ In general, it would seem that on this view, virtual objects have both virtual and non-virtual shapes and colors. They have the shapes and colors that normal perceivers would experience in normal viewing conditions (non-virtual shapes and colors) and those normal perceivers would experience in viewing conditions normal for the given virtual reality (virtual shapes and colors).
} 
shirt; maybe the former looks red only in the way virtual objects do. And it is fine if the same is so for the roundness of my virtual belly. But these virtual objects do appear to at least have shapes and sizes, where this isn't a matter of mere polysemy. And so whether virtual realism is true and experiences in virtual reality are generally nonillusory depends on there being a sense in which virtual colors and shapes and non-virtual colors and shapes are in a sense the same or similar kinds of features. But is this so?

\section{A challenge for phenomenal spatial functionalism}

I will start by noting that in order to establish a similarity or sameness in kind between virtual and non-virtual properties, one need not establish phenomenal functionalism about these propertiesthere just must be some basis for similarity between virtual shapes and colors and non-virtual shapes and colors. But given that virtual objects (guns, shirts, bellies) are constituted out of different materials than their non-virtual counterparts, one might wonder how they could bear the same or similar kinds of properties. This is where functionalism comes in. Just as in the philosophy of mind, where the fact that humans made of cells and artificial intelligences made out of silicon could both have mental states prompted Hilary Putnam (1967) and others to propose that what makes something an object bearing psychological features is not that it is made out of a certain kind of stuff but rather the kind of causal role the object is capable of playing, so the phenomenon of multiple realization leads us towards a similar kind of functionalism for the features of virtual realities. And so, in the present discussion, we can note that even if virtual and non-virtual shirts may be made out of very different kind of materials (silicon and cotton, respectively), they can be similar, at least in the respect that in the right kinds of circumstances, they both cause red experiences in normal perceivers.

But while phenomenal functionalism may be an orthodox position among philosophers of color, this is a much more questionable position to hold about spatial properties. Philosophers from at least as far back as Democritus have recognized a distinction between those properties objects have in virtue of the responses they produce in observers and those that objects have in themselves independent of what 
observers will experience. Spatial properties like shapes and sizes are typically thought to be paradigmatic examples of the latter. This is encapsulated in Locke's primary/secondary quality distinction. In taking phenomenal functionalism to be true not only of color but also of spatial properties, Chalmers collapses this long-standing distinction.

That this distinction has been historically influential does not mean it is correct. As a naturalist, I believe one ought to look at our best scientific theories to discover what is the right way to think of the features of the objects around us. The science of color is to a large extent a science of the kinds of experiences light reflected by or off of the surfaces of objects may cause in perceivers. And there are many reasons to see why this is so. Let me just note one that will serve to make a case for the difference between color and spatial properties. ${ }^{2}$ This is the phenomenon of metamerism, that objects with quite diverse surface structures and spectral reflectance profiles may all cause the same kind of color experiences in normal viewing conditions. Despite differences in their objective structure, metameric objects are generally classified as of the same color, not as different. This suggests that color features track similarities that are at least partly subjective, that is, based on the character of observers' phenomenal experiences, and that facts about these phenomenal similarities trump facts about objective resemblance when deciding which objects are or are not the same color.

These considerations do not apply to space and spatial features. To begin, our best science of space is not a science of what objects and their surfaces may cause in perceivers but of what objective structures there are in reality. Our best science of space is general relativity, a theory standardly interpreted as describing a spacetime manifold characterized by certain objective symmetries. One might try to push on this a little in the following way to motivate phenomenal functionalism. One might note that relativity shows us that objects actually don't have their spatial features absolutely. What is absolute rather are various spatiotemporal relations. Facts about spatial distances (and so facts about the lengths, sizes, and shapes of objects), according to relativity, obtain only relative to individual frames of 1983).

${ }^{2}$ One can find very many others in the color literature (for example, Rossotti 
reference, which may be observers' frames of reference, facts about how fast someone is moving. But this would not be a successful way of motivating phenomenal functionalism, for even if spatial features were observer-dependent according to relativity theory, this would not make spatial features dependent on observers' experiences as opposed to dependent on objective features about their states of motion. The former is what phenomenal functionalism requires.

Moreover, we may discuss spatial cases that are analogous to the phenomenon of metamerism, and when we do, we reach an opposite conclusion than that found for the case of color. This time, we consider situations in which two objects are constituted out of very different kinds of objective structures and yet produce the same spatial experiences in observers. Optical engineers can produce objects that deceive observers about their spatial features. For example, paraxial cloaks can bend light around making objects look hollow in ordinary viewing conditions (Choi and Howell 2014). These exotic kinds of objects make the point, but to do so we only need appeal to mundane facts with which anyone who has used clothing to make oneself appear taller or thinner is well aware. Unlike what we saw for the case of color metamers, the facts about what shape or size an object is does not track what experiences these objects cause, but rather their intrinsic structures. I may trick people into appearing as tall as my five foot eight friend by wearing higher-waisted pants, but the pants won't actually make me any taller. This again would seem to indicate that phenomenal functionalism, which may seem plausible with respect to colors, is not similarly plausible with respect to spatial features.

If one takes this view, what is the more common view, that phenomenal functionalism is much less promising for spatial properties than for colors, then this means that although one can use phenomenal functionalism about virtual and non-virtual properties to argue that virtual objects do have the colors they appear to have, one cannot use it to similarly argue that virtual objects have the spatial properties (e.g. the lengths, shapes, and sizes) they appear to have, since they appear to have similar spatial properties to those of their nonvirtual counterparts. And this undermines Chalmers's argument for that part of virtual realism that says that experiences in virtual reality are generally non-illusory. He can use the argument in his paper 
to motivate the claim that virtual objects have some of the properties they appear to have, but not all.

Be that as it may, this doesn't mean the case for virtual realism is closed. For Chalmers could instead adopt an alternative view about the nature of spatial features and then use this view to motivate a similarity between the spatial properties of virtual and non-virtual objects. For example, one very common view is that spacetime manifolds are constructed out of certain symmetry structures (e.g. Esfeld and Lam 2008). Such symmetry structures could be equally present in virtual and non-virtual settings, thus grounding similarities between the spatial properties of virtual and non-virtual objects. And this is because the possession of symmetries doesn't depend on the material composition of an entity, but rather its more abstract structure. In his paper, Chalmers proposes phenomenal functionalism as an account of spatial properties, and I believe it is a theory of spatial properties he is sympathetic with, but the overall argument in this part of the paper doesn't really hang on that controversial account of spatial properties, so long as there is some alternative account that would support a similarity between the spatial properties of non-virtual objects and the spatial properties of their virtual counterparts, one that can account for the fact that these objects appear to have similar features.

Unfortunately I don't think this more common, symmetry-based view about the nature of spatial properties will help get Chalmers what he wants, since although it is possible that virtual objects and their non-virtual counterparts share symmetry features, there is no reason why this would have to be so. And indeed in most cases, it is reasonable to expect that the digital object constituting, say, a virtual baseball will not share symmetry features with its non-virtual counterpart. But to say this is not to say that Chalmers could not appeal to some other third account of spatial features that would underwrite a similarity in kind between virtual and non-virtual shapes and sizes. I just don't know what that account would be.

\section{A place between fiction and (non-virtual) reality}

Partially to reinforce how much I find phenomenal functionalism unhelpful here, I now want to make the case that even to the extent that 
phenomenal functionalism may be a plausible view about both the colors and spatial features of non-virtual objects, there are reasons to doubt this may be the correct view about the colors and spatial features of virtual objects. I will describe some cases, and there is a larger point that may be drawn from them. This is that there may be facts about what is true in the virtual reality that may trump other facts about features of the virtual reality setting when it comes to establishing the color and spatial properties of virtual objects, including facts about what normal perceivers experiences in circumstances that are normal for the virtual reality and facts about objective symmetries. In this respect, the facts about the properties objects have in virtual realities may be determined in a way that is more akin to the way facts are settled about the properties objects have in fictions, rather than in non-virtual realities.

Let me just use a simple case building on one we have already considered. As I mentioned, the default avatar in Gun Club VR appears to have a round belly. Looking down when you play the game, this makes it hard to see and access the ammunition and other tools on your avatar's belt. But suppose as you start the game, the following dialogue appears in front of you:

Your character in Gun Club is that of a skinny police rookie posing as a burly gun aficionado in order to foil a notorious criminal gang. Use this shooting range to develop your gun skills and perfect your cover.

In the game, you will always appear round to normal perceivers in viewing conditions normal for the virtual reality. But your avatar (and his belly) are not virtually round. There are facts about what is true in the virtual reality that trump facts about any appearances and these include that your avatar's belly is flat, not round.

A similar conclusion follows if, as was suggested in the previous section, we reject phenomenal functionalism about spatial properties and instead endorse a symmetry-based structuralism. Is your avatar skinny or is he round? The truth seems to be that he is, despite appearances, skinny. But this is not grounded in any facts about the symmetries of objects in the virtual reality. For there is no skinny object present in the virtual reality. After all, what reason would there be for those who designed Gun Club VR to program a skinny 
body underneath the bulky red shirt or to use a skinny digital object to realize your avatar? Again, there are facts about what is true in the virtual reality that trump any structural features of the objects in it.

It is easy enough as well to extend this case to one in which there are facts about the colors of virtual objects determined by bits of dialogue that again trump facts about what normal perceivers will experience in viewing settings that are normal for the virtual reality, or indeed (were one to be skeptical about phenomenal functionalism about colors) that trump facts about the intrinsic structures of virtual objects. Suppose instead the opening dialogue said:

Your character in Gun Club is that of a pale police rookie posing (using clever optical tricks) as a weather-beaten gun aficionado in order to foil a notorious criminal gang. Use this shooting range to develop your gun skills and perfect your cover.

The fact of the matter is that your avatar is pale, but he will always appear to those in the game as tanned. Moreover, there is no reason for the game designers to program a pale man and complicated lighting. The physical features of the avatar himself will suggest he is tanned, not pale.

What this suggests to me is not that virtual objects are not real, nor that virtual events don't really take place. It suggests that experiences in virtual reality may not always be veridical, but this is not a problem for virtual realism, which only needs the claim that they are generally veridical. Although I don't see a challenge here for virtual realism, or at least a version of virtual realism slightly modified from how Chalmers states the position (one requiring only general veridicality), these sorts of cases make me worry that the line between virtual realism and virtual fictionalism is less sharp than Chalmers suggests in his paper. For in non-virtual reality, the shapes and colors of objects do not get decided by bits of dialogue. This is something that is perfectly commonplace in fiction, but not in non-virtual reality. So, I would like to know how Chalmers would accommodate these cases and whether they require a view according to which virtual reality occupies a kind of middle ground in between fiction and non-virtual reality.

An initial concern one might raise about the point I am making in this section is that I may be putting too much emphasis on contingent 
features of virtual reality video games in which there are fictions embedded in the games. Chalmers warns of this in his paper:

There is of course a close connection between any role-playing game and an associated fiction, but this connection holds whether the game is virtual or non-virtual. If a human in physical reality plays the role of Gandalf casting a spell in Middle Earth, the event of Gandalf casting a spell is fictional, but the underlying bodies and movements are real. Likewise, if an avatar in virtual reality plays the role of Gollum stealing the ring, the event of Gollum stealing the ring is fictional, but this is consistent with the underlying avatars and movements within the virtual realm being real. (Chalmers 2017: 316)

So, one might try to respond that in these cases, my avatar is round, although there is a fiction in the game according to which he is skinny; and my avatar is tanned, though there is a fiction in the game according to which he is pale. And so, one might try to argue, the facts about what experiences are caused or the physical structure of digital objects is what determines which colors and shapes these virtual objects are; the dialogue only determines fictions within the game. But this seems backwards. In the cases I describe in this section, there are fictions within the virtual realities, but the fictions are that the avatar is round or tanned. The facts about the virtual objects in the game, what I concede are real digital objects are, however, skinny and pale.

\section{Conclusion}

What I am arguing, then, is that although Chalmers has a good case for the reality of virtual objects and could conceivably make the case for virtual objects' generally having the properties they seem to have, particularly were he to find a more plausible view about the spatial properties of non-virtual objects, there is also a way in which virtual objects are different from non-virtual objects that makes them in a sense more like fictional objects. This is that their colors and spatial properties may sometimes be determined by facts that go beyond how they appear to observers or objective facts about their physical constitution. $^{3}$

\footnotetext{
${ }^{3}$ Thank you to David Yates for inviting me to take part in this symposium.
} 


\section{Alyssa Ney \\ University of California, Davis aney@ucdavis.edu}

\section{References}

Chalmers, David. 2012. Constructing the World. Oxford: Oxford University Press.

Chalmers, David. 2017. The virtual and the real. Disputatio 9(46): 309-52.

Choi, Joseph S.; and John C. Howell. 2014. Paraxial ray optics cloaking. Optics Express 22(24): 29465-78.

Esfeld, Michael; and Vincent Lam. 2008. Moderate structural realism about space-time. Synthese 160(1): 27-46.

Putnam, Hilary. 1967. Psychological predicates. In Art, Mind, and Religion, ed. by W.H. Capitan and D.D. Merrill. Pittsburgh: University of Pittsburgh Press, 37-48.

Rossotti, Hazel. 1983. Colour: Why the World Isn't Grey. Princeton: Princeton University Press. 